\title{
PHYLOGENETIC DIVERSITY OF MICROORGANISMS ASSCOCIATED WITH THREE MARINE SPONGES FROM MIEN TRUNG SEA OF VIET NAM
}

\author{
Nguyen Thi Kim Cuc ${ }^{1, *}$ Ton That Huu Dat ${ }^{2}$, Tran Thi Hong ${ }^{2}$, Pham Viet Cuong ${ }^{1,2}$ \\ ${ }^{1}$ Institute of Marine Biochemistry, VAST, 18 Hoang Quoc Viet, Cau Giay, Ha Noi \\ ${ }^{2}$ Mien Trung Institute for Scientific Research, VAST, 321 Huynh Thuc Khang, Hue \\ "Email: kcnguyenthi@gmail.com
}

Received: 27 October 2016; Accepted for publication: 24 November 2016

\begin{abstract}
Using culture - independent technique, hypervariable V4 region of 16S rDNA library sequencing by MiSeq, the baterial communities of three host sponges Rhabdastrellasp. DN, Spheciospongiasp. QT and Clathriasp. NT from Mien Trung sea were characterized. The phylogenetic analysis showed that bacterial community structures of the three investigated sponges similar to each other regarding 10 common phyla, although abundance of these phyla was different for each sponge. Phylum Thaumarchaeota was rich component for three sponges, especially in NT sponge (31.89\%). In this sponge, 3 phyla Planctomycetes, Verrucomicrobia and Firmicutes were undetected in other 2 sponge samples. Phyla Cyanobacteria was observed only in DN sponge.

The obtained amplicons were assigned in different taxonomic levels (class, order, family and genus) based on Silva database. At class level, Gammaproteobacteria was abundant in three sponges; and Caldilineae, Marine-group I were dominant in DN; mealwile, in QT other dominant classes were Marine-group I and Betaproteobacteria. For NT, they were Cytophaga and Deferribacteres. In general, all three sponges harbored abundant and genetically diverse microbial associated consortia and they shared several common bacterial operational taxonomic units, although with different abundance.
\end{abstract}

Keywords: MiSeq sequencing, phylogenetic diversity, sponge associated microorganism, 16S rRNA, V4 region.

\section{INTRODUCTION}

Microbes play fundamental roles in the functioning of most ecosystems. Currently, it is calculated that land and ocean have $3.6 \times 10^{29}$ and $4-5 \times 10^{30}$ microbial cells, respectively. The main method for determination of phylogeny and so quantification of microbial biodiversity is $16 \mathrm{~S}$ rRNA sequences for prokaryotes and 18S rRNA for eukaryotes [1].

Sponges (phylum Porifera) are oldest sessile, filter-feeding inverte brates that in habit diverse marine ecosystems. They are known to host large microbial community, accounting for up to $50 \%$ of sponge tissue volume and this community is specific for sponges [2,3]. In recent years, many researches used molecular culture-independent techniques for survey of sponge- 
associated microorganisms's diversity in different marine ecosystems. Studies based on cultureindependent 16S ribosomal RNA gene sequence (16S rDNA) analyses have provided insights into microbial communities in terms of composition. Still, many important questions in microbialecology remain unsolved and have been awaiting technological progress to be investigated. The advent of next generation sequencing technologies is enabling the exploration of microbial diversity at an unprecedented scale. Illumina 16S rDNA reads as short as $100 \mathrm{bp}$ can be enough for an accurate taxonomic characterization of microbial communities [4]. But read-length limitations can be overcome with the introduction of newer. Illumina sequencers that produce longer reads, for example, MiSeq sequencer produce $2 \times 250$ bp reads, which after merging cangenerate reads up to $490 \mathrm{bp}$ [5].

The goal of this paper was assessed diversity of microorganisms associated with three marine sponges collected from Mien Trung sea by analyzing hypervariable V4 region of $16 \mathrm{~S}$ rDNA gene after MiSeq sequencing.

\section{MATERIALS AND METHODS}

\section{Sponge samples}

Sponges collected from Mien Trung sea by SCUBA at a depth $10-15 \mathrm{~m}$ and were identified as Rhabdastrellasp.DN (Lang Co); Spheciospongia sp.QT (Cua Tung, Quang Tri); Clathriasp.NT (NhaTrang Bay). Sponges were put in sterile plastic bottles with $30 \%$ glycerol on ice, transferred to laboratory and stored at $-20{ }^{\circ} \mathrm{C}$ for further investigation.

\section{Extraction of total DNA}

Sponge samples were washed 3 times with sterile artificial sea water and then $10 \mathrm{~g}$ of sample were cut into pieces, ground to uniform in TE buffer, $\mathrm{pH} 8(10 \mathrm{mM}$ Tris- $\mathrm{HCl}, 1 \mathrm{mM}$ EDTA). Filtered the mixture through 2 layers of muslin and centrifuged at $250 \mathrm{~g}$ for 1 minute for removing sponge debris. Cells were collected by centrifugation of the supernatant at $8000 \mathrm{~g}$ for 15 minutes at $4{ }^{\circ} \mathrm{C}$. Cells were washed by TE 50 solution $(10 \mathrm{mM}$ Tris- $\mathrm{HCl}, 50 \mathrm{mM}$ EDTA, pH 8). Total DNA was extracted by E.Z.N.A ${ }^{\circledR}$ Soil DNA Kit, according to the manufacture's instruction.

\section{S rDNA MiSeq library construction}

For diversity's assessment of sponge associated microorganisms, analyzing of V4 hypervariable region of $16 \mathrm{~S}$ rDNA was used. In first PCR, the V4 region (about $300 \mathrm{bp}$ ) of $16 \mathrm{~S}$ rDNA was amplified using 515F (GTGYCAGCMGCCGCGGTAA) and 806R (GGACTACNVGGGTWTCTAAT) primers [6]. Reaction was performed in a final volume of $25 \mu \mathrm{l}$ containing $5 \mu \mathrm{l}$ Green HF buffer $5 \mathrm{x} ; 200 \mu \mathrm{M}$ of each of the four dNTPs (ThermoFisher); $0.2 \mu \mathrm{M}$ of each primer; $0.25 \mu 1$ Phusion Hot start DNA polymerase $2 \mathrm{U} / \mu 1$ (ThermoFisher) and 1 $\mu 1$ DNA template. The amplification program was: initial denaturation for $5 \mathrm{~min}$ at $98{ }^{\circ} \mathrm{C} ; 25$ cycles of ( 45 seconds at $98{ }^{\circ} \mathrm{C} ; 30$ seconds at $50{ }^{\circ} \mathrm{C} ; 30$ seconds at $72{ }^{\circ} \mathrm{C}$ ); extension for $10 \mathrm{~min}$ at $72{ }^{\circ} \mathrm{C}$, kept at $4{ }^{\circ} \mathrm{C}$. PCR products were checked on $1.8 \%$ agarose gel.

The second PCR was carried out in order to ligate of barcodes into PCR products obtained in the first PCR. Every sample has its own barcode. Forward barcodes were CCAAGTCA; GAGTTCATACT; and TCAGGCGAT for DN, QT and NT, respectively. Reverse barcodes were CCAAGTCA; GAGTTCATTCA and TCAGGCGAA for DN, QT and NT, respectively. The final volume of the reaction was $31 \mu \mathrm{l}$ containing $10 \mu \mathrm{l}$ of Green HF buffer $5 \mathrm{x} ; 200 \mu \mathrm{M}$ of each of the four dNTPs; $0.5 \mu \mathrm{M}$ of each barcode and $0.5 \mu \mathrm{l}$ Phusion Hot start DNA polymerase 
$2 \mathrm{U} / \mu \mathrm{l}$ (ThermoFisher); PCR product as template from $1^{\text {st }}$ PCR $2.5 \mu$. The amplification program was: initial denaturation at $98{ }^{\circ} \mathrm{C}$ for $1 \mathrm{~min} ; 5$ cycles (10seconds at $98{ }^{\circ} \mathrm{C} ; 20$ seconds at $52{ }^{\circ} \mathrm{C}$; 20 seconds at $72{ }^{\circ} \mathrm{C}$ ); extension for 10 minutes at $72{ }^{\circ} \mathrm{C}$, kept at $4{ }^{\circ} \mathrm{C}$. PCR products were checked on $1.8 \%$ agarose gel.

The PCR products after second PCR were purified by HighPrep ${ }^{\mathrm{TM}}$ PCR protocol-MagBio kit and concentration was calculated by Qubit kit (Invitrogen). Equal amount (150 $\mu \mathrm{g})$ of purified PCR product from each sample was pooled for library creation. The formed library was purified by HighPrep ${ }^{\mathrm{TM}}$ PCR protocol-MagBio kit and was sequenced by MiSeq sequencer.

\section{Analysis 16S rDNA library}

The 16S rDNA library after sequencing was analyzed by Qiime Virtual Box 1.9.0 with the QIIme 1.3.0 pipeline (http://qiime.sourceforge.net/). First, reads without barcodes or noncomplementary with primers were removed from the library. Once trimmed and assigned to samples, sequences were processed using the Qiime's UCLUST method in order to cluster the sequences in operational taxonomic units (OTUs) at the $98.5 \%$ identity level. OTUs with abundance less than $0.1 \%$ of total reads were also eliminated. The most abundant sequence of each OTU was selected as representative sequence and subsequently aligned using PyNAST [7] against Silva database. Possible chimeric sequences were identified using Qiime's Chimera Slayer and subtracted from the previous generated OTU list, generating a non-chimeric nonredundant OTU list. The taxonomic affiliation was assigned to each OTU using the Ribosomal Data Project (RDP) Classifier at a confidence threshold of $90 \%$ [8].

\section{RESULTS}

\section{Amplification of $\mathrm{V} 4$ region of $16 \mathrm{~S}$ rRNA and barcodes ligation}

The V4 region of 16S rRNA gene was amplified according above mentioned method. PCR products were checked on $1.8 \%$ agarose gel (Fig. 1) and were about $300 \mathrm{bp}$ as calculated theoretically.

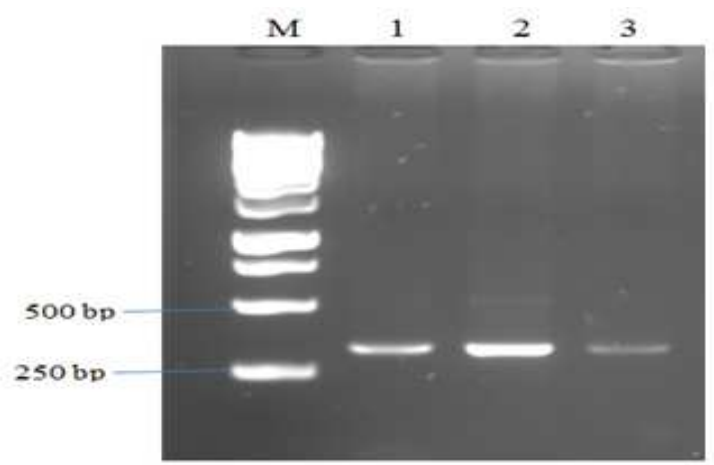

Figure 1. PCR products of V4 region of sponge associated bacterial 16S rDNA. Lanes 1, 2, 3: DN, QT, NT, respectively. M: Marker DNA $1 \mathrm{~kb}\left(\right.$ GeneRuler $\left.^{\mathrm{TM}}\right)$.

For barcodes ligation into V4 region of 16S rRNA, PCR products of first PCR were used as template in second PCR and the reaction was performed as described. PCR products of $2^{\text {st }}$ round PCR were checked on $1.8 \%$ agarose gel (Fig. 2). 


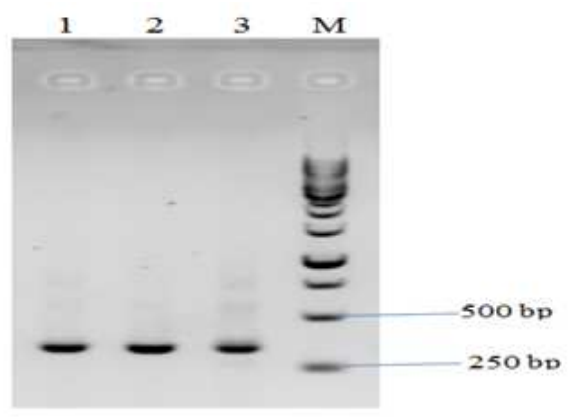

Figure 2. PCR products after barcodes ligation. Lanes 1, 2, 3: DN, QT and NT, respectively. M: marker DNA $1 \mathrm{~kb}\left(\right.$ GeneRuler $\left.^{\mathrm{TM}}\right)$.

\section{Composition of bacterial communities}

Sequenced 16S rRNA amplicons were rigorously assessed for quality, as well as contaminant and putative chimeras. The result showed that $55.9 \%$ of reads from sponge DN (corresponding to 264550 reads), $58.29 \%$ of reads from sponge QT (corresponding to 45965 reads), and $49.61 \%$ of reads from sponge NT (corresponding to 70994 reads) meet the requirements. These reads were grouped into 125, 117 and 132 OTUs for DN, QT and NT, respectively, defined by $98.5 \%$ or greater sequence similarity.

Based on Project (RDP) Silva database v.1.1.1, the received OTUs were identified to phylum, class, order, family and genus levels.

Table 1. Composition of bacterial communities in three sponge species.

\begin{tabular}{|l|c|l|c|l|l|}
\hline \multicolumn{2}{|c|}{ DN } & \multicolumn{2}{c|}{ QT } & \multicolumn{2}{c|}{ NT } \\
\hline Phyla & $\begin{array}{l}\% \text { total } \\
\text { reads }\end{array}$ & \multicolumn{1}{c|}{ Phyla } & $\begin{array}{l}\% \text { total } \\
\text { reads }\end{array}$ & \multicolumn{1}{c|}{ Phyla } & $\begin{array}{l}\% \text { total } \\
\text { reads }\end{array}$ \\
\hline Thaumarchaeota & 10.68 & Thaumarchaeota & 16.84 & Thaumarchaeota & 31.89 \\
\hline Acidobacteria & 2.45 & Acidobacteria & 4.14 & Acidobacteria & 20.17 \\
\hline Actinobacteria & 1.99 & Actinobacteria & 7.02 & Actinobacteria & 15.43 \\
\hline Bacteroidetes & 3.21 & Bacteroidetes & 1.92 & Bacteroidetes & 7.65 \\
\hline Chloroflexi & 29.93 & Chloroflexi & 8.15 & Chloroflexi & 7.19 \\
\hline Deferribacteres & 0.69 & Deferribacteres & 0.13 & Deferribacteres & 7.01 \\
\hline Gemmatimonadetes & 10.59 & Gemmatimonadetes & 7.43 & Gemmatimonadetes & 1.13 \\
\hline Nitrospirae & 4.55 & Nitrospirae & 1.82 & Nitrospirae & 1.05 \\
\hline Proteobacteria & 32.14 & Proteobacteria & 51.47 & Proteobacteria & 0.87 \\
\hline Spirochaetes & 0.12 & Spirochaetes & 0.83 & Spirochaetes & 0.78 \\
\hline Cyanobacteria & 1.52 & & & Planctomycetes & 0.87 \\
\hline & & & & Verrucomicrobia & 0.41 \\
\hline & & & & Firmicutes & 5.18 \\
\hline Unclassified & 2.11 & Unclassified & 0.23 & Unclassified & 0.33 \\
\hline
\end{tabular}


The data in the Table 1 showed that sponge associated bacterial communities exhibited high diversity, comprising 10 common phyla for 3 investigated sponge samples. But abundance of phyla was different for each sponge. Sequences affiliated with Proteobacteria were dominant in DN and QT (32.14 \% and $51.47 \%$, respectively), but in NT, it was only $0.87 \%$. Thaumarchaeota-affiliated sequences comprised an abundant component of microbial communities in all three host species (>10\% total reads), especially in NT, it occupied about 32 $\%$ of total reads. Among the three host sponges, NT exhibited more richness in bacterial communities than DN and QT. Three phyla (Planctomycetes, Verrucomicrobia, Firmicutes) observed in NT were obsent in DN and QT, but Cyanobacteria was discovered only in DN.

Based on Silva database, the reads with class assignments, it was observed that Gammaproteobacteriawas dominant in all three sponge samples; although Betaproteobacteria was given about $15 \%$ of total reads in QT, in DN it was only $2.80 \%$ and absent in NT sponge. Marine group I was occupied more than $10 \%$ of total reads in DN and QT, but in NT this group was minor component. Meanwhile, Cytophagia and Deferribacteres were dominant classes in NT, but in QT and DN were in small numbers. The Deltaproteobacteria was present in all investigated sponges with similar proportion.

The obtained reads were also assigned to families and sponge QT had most (26 families), next NT composed 21 families and DN had least, only 19 families. At family level, the abundance of Caldilineaceae was decreased as DN > QT > NT; Rhodothermaceae was opposite as NT > DN > QT and Halellaceae was dominant in QT, minor in NT and absent in DN sample (Fig. 3). Sponge QT haboured 5 specific families Shewannellaceae, Pseudomonadaceae, Flammeovirgaceae, Acidobacteriaceae and Kordiimonadaceae; sponge NT composed 3 specific families Puniceicoccaceae, Hyphomonadaseae and Alteromonadaceae, which absent in other two remaining sponges.
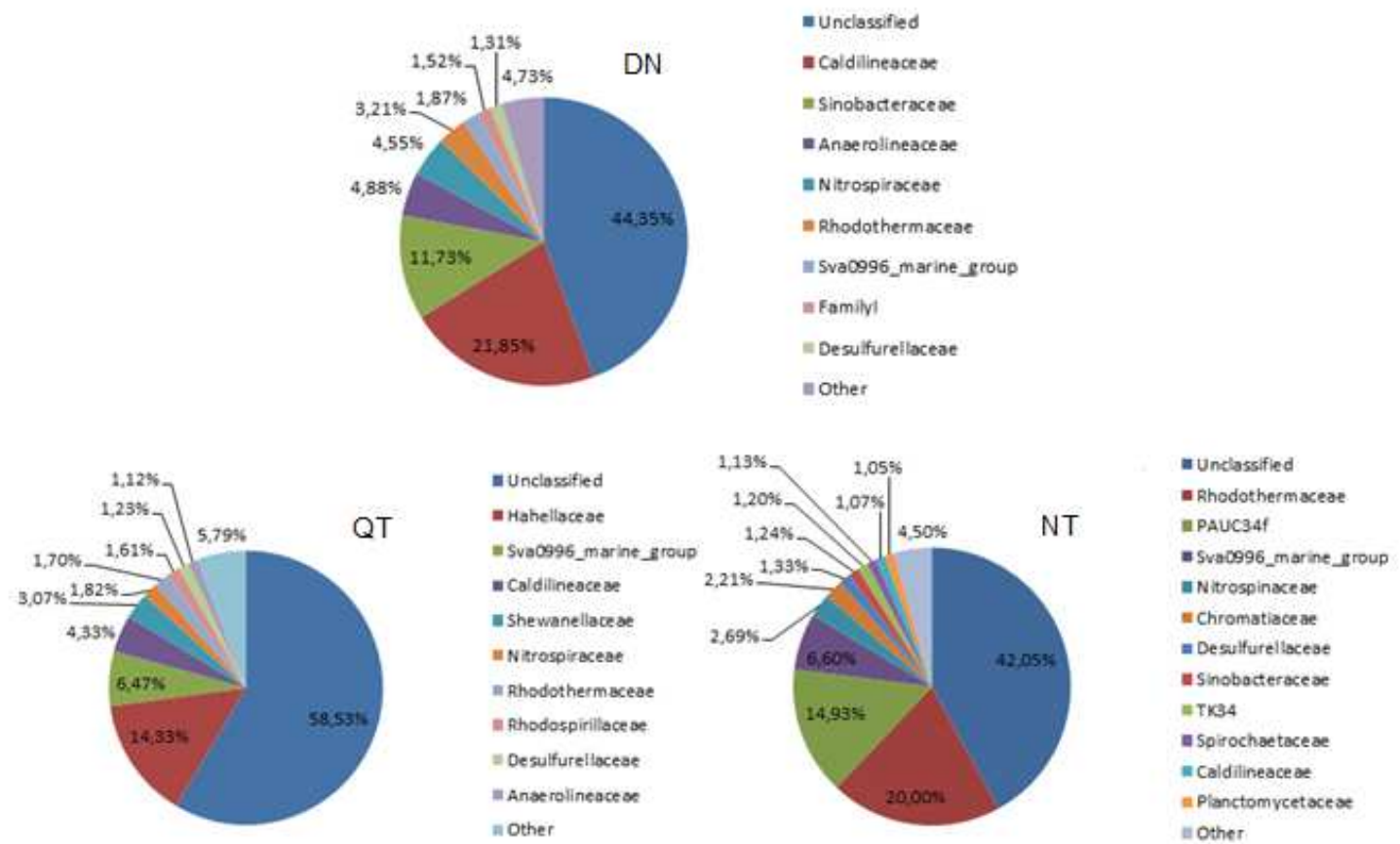

Figure 3. Family-level taxonomic assignments of 16S rRNA sequences for 3 sponges. 
Table 2. Assignment of the reads to genera.

\begin{tabular}{|l|l|l|l|l|l|}
\hline \multicolumn{5}{|c|}{ GENERA } & \multicolumn{2}{c|}{ QT } \\
\hline Name & $\%$ total reads & Name & $\begin{array}{l}\% \text { total } \\
\text { reads }\end{array}$ & Name & $\begin{array}{l}\% \text { total } \\
\text { reads }\end{array}$ \\
\hline Unclassified & 71.31 & Unclassified & 72.07 & Unclassified & 89.51 \\
\hline Caldilinea & 21.45 & Endozoicomonas & 13.94 & Nitrosococcus & 2.21 \\
\hline Nitrospira & 4.55 & Caldilinea & 4.33 & Candidatus_Entotheonella & 2.05 \\
\hline Nitrosococcus & 0.92 & Shewanella & 3.07 & Caldilinea & 1.07 \\
\hline Rhodovulum & 0.63 & Nitrospira & 1.82 & Rhodopirellula & 1.05 \\
\hline Defluviicoccus & 0.49 & Defluviicoccus & 1.61 & Spirochaeta & 1.03 \\
\hline Granulosicoccus & 0.24 & Pseudomonas & 0.92 & Cerasicoccus & 0.87 \\
\hline Pseudospirillum & 0.17 & Spirochaeta & 0.38 & Nitrospira & 0.87 \\
\hline Spirochaeta & 0.12 & Acinetobacter & 0.35 & Pseudospirillum & 0.65 \\
\hline Bdellovibrio & 0.11 & Bdellovibrio & 0.30 & Defluviicoccus & 0.48 \\
\hline & & Aeromonas & 0.26 & Rhodovulum & 0.19 \\
\hline & & Granulosicoccus & 0.23 & & \\
\hline & & Persicobacter & 0.22 & & \\
\hline & & Pseudovibrio & 0.14 & & \\
\hline & & Ruegeria & 0.13 & & \\
\hline & & Rhodovulum & 0.11 & & \\
\hline
\end{tabular}

The reads were also assigned to genera (Table 2). The obtained results showed that in DN, genus Caldilinea was dominant (more than $21 \%$ of total reads), Nitrospira $(4.55 \%)$ and 7 other genera, each with less $1 \%$ total reads. Genus Endozoicomonas was the main group in QT (approximaterly $14 \%$ total reads); Caldilinea $4.33 \%$; Shewanella $3.07 \%$; Nitrospira $1.82 \%$; Defluviicoccus $1.61 \%$ and 11 other genera each with less $1 \%$ total reads. NT composed 10 identified genera, among them Nitrosococcus was $2.21 \%$; Candidatus-Entotheonella $2.05 \%$; followed by Caldilinea $1.07 \%$; Rhodopirellula $1.05 \%$; Spirochaeta $1.03 \%$ of total reads. It was obviously that three sponge samples share some common genera, while they possess their own genera, which were specific for them, although in small percentage of total reads.

\section{DISCUSION}

Amplicon sequencing, in particular that of the small subunit rRNA gene (16S rRNA gene in Bacteria), is a widely applied approach to study the composition, organization and spatiotemporal patterns of microbial communities, due to its ubiquity across all domains of life $[9,10]$. Currently, illumina is the state of the art when it comes to16S rRNA gene amplicons [11]. Identification and characterization of rRNA genes help in the analysis of phylogeny and quantification of microbial diversity [12] and various communities have been studied using metagenomic approach. By amplification of V4 region of 16S rRNA of several Antarctic 
sponges, Rodrisguez-Marconi et al. (2015) found that dominant phyla were Proteobacteria, followed by Bacteroidetes, Verrucomicrobia, Thaumarchaeota and Planctomycetes [13] Proteobacteria are common in marine environments and are always associated with marineplants or animals [14]. The phylum Proteobacteriahave been found in different sponges from the same or different geographic location, for example, Aplysinacavernicola, Rhopaloeidesodorabile, Theonellaswinhoei, Halichrondriapanicea, and the sponges Jaspisjohnstoni and Plakortislita. Proteobacteria have been suggested to have varied effects on sponge hosts and have been proposed that they are in close symbiotic relationship with sponges [15]. Based on received result, this phylum is present in all three our sponges, although with different proportions.

Thaumarchaeota represent a unique phylum within the domain Archaea that embraces ammonia-oxidizing organisms from soil, marine waters, and hot springs [16]. Thaumarchaeota were detected in different abundances in many other marine sponges, including Arctic and Irish deep-sea environments $[17,18]$. This phylum was discovered in all three our sponges with high proportion, especially in NT (about $32 \%$ total reads). The vast majority of phyla discovered in DN, QT and NT were also reported for other marine sponges [19].

Little is known about the $S$. vespatium and Clathriareinwardtii microbial communities. Concerning micro-biodiversity of $R$. globostellata, Schmissusing high-throughput-sequencing amplicon screening detected 16 bacterial phyla associate with $R$. globostellata [20]. In our sample (DN), cyanobacteria was detected, but only 11 phyla were identified. It was known that the abundant microbial phyla in $R$. globostellata (i.e., Acidobacteria, Chloroflexi, Cyanobacteria, and Gemmatimonadetes) are known to contain (bacterio) chlorophyll-based phototrophic lineages [21], it is possible that photoheterotrophic bacteria also play a considerable role in this host-specific microbiota. Another dominant phylum Chloroflexi present in all our samples and distributed in sponges from different depths suggests that they may not be phototrophically active within the sponges, although their function in sponges is yet unclear [22].

Environmental factors specific to distinct habitats may play a role in structuring symbiont communities and host factors specific for each sponge species may also influence the composition of symbiotic bacterial communities in sponges [19], but the work of Blanquersupporthost-related, evolutionary features rather than environmental conditions, as the maincause shaping the structure of the sponge microbial communities [23]. Our results showed that bacterial communities in three sponge species collected in different locations were dissimilar in all classification levels, suggesting that both environmental conditions and host specificity affect composition of bacterial communities in sponges.

\section{CONCLUSION}

The diversity of microorganisms associated with 3 sponges collected from Mientrung sea of Vietnam was evaluated. Obtained results after analyzing of 16s rRNA library showed that all three sponge samples harbored the same main taxonomic groups of microbes, but possessed particular groups. This work shows that even when sponges sharing an important part of the microbial communities with each other, sponge-associated microbes are characterized by both specialists and generalists.

Acknowledgements. This research was supported by the Vietnamese Government project in cooperation with The Netherlands, code ĐTĐLCN.17/14 of Ministry of Science and Technology. 


\section{REFERENCES}

1. Ferrer M., Beloqui A.,Timmis K. N., Golyshin P. N. - Metagenomics for Mining New Genetic Resources of Microbial Communities, J. Mol. Microbiol. Biotechnol 16 (2009) 109-123.

2. Hill M., Hill A., Lopez N., Harriott O. - Sponge-specific bacterial symbionts in the Caribbean sponge, Chondrillanucula (Demospongiae, Chondrosida), Marine Biology 148 (2006) 1221-1230 DOI 10.1007/s00227-005-0164-5.

3. Wang G. - Diversity and biotechnological potential of the sponge-associated microbial consortia, J. Ind. Microbiol. Biotechnol. 33 (2006) 545-551.

4. Li Z., Hu Y., Liu Y., Huang Y., He L., Miao X. - 16S rDNA clone library-based bacterial phylogenetic diversity associated with three South China Sea sponges World J. Microbiol. Biotechnol. 23 (2007)1265-1272 DOI 10.1007/s11274-007-9359-x.

5. Logares R., Sunagawa S, Salazar G, Cornejo-Castillo FM, Ferrera I, Sarmento H, Hingamp P, Ogata H, de Vargas C., Lima-Mendez G., Raes J., Poulain J., Jaillon O., Wincker P., Kandels-Lewis S., Karsenti E., Bork P., Acinas S.G. - Metagenomic 16S rDNA Illumina tags are a powerful alternative to amplicon sequencing to explore diversity and structure of microbial communities. Environ. Microbiol. 16(9) (2014) 2659-71. doi: $10.1111 / 1462-2920.12250$.

6. Caporaso J. G., Lauber C. L., Walters W. A., Berg-Lyons D., Huntley J., et al. - Ultrahigh-throughput microbial community analysis on the Illumina HiSeq and MiSeq platforms. The ISME Journal 6 (2012) 1621-1624. doi: 10.1038/ismej.2012.8 PMID: 22402401.

7. DeSantis T. Z., Hugenholtz P., Keller K., Brodie E. L., Larsen N., Piceno Y. M., Phan R., and Andersen G. L. - NAST: A multiple sequence alignment server for comparative analysis of 16S rRNAgenes, Nucleic Acid Res. 34 (2006)W394- 399.

8. Cole J. R., Wang Q., Fish J. A, Chai B., McGarrell D. M., Sun Y., Brown C. T., PorrasAlfaro A., Kuske C. R. and Tiedje J. M. - Ribosomal Database Project: data and tools forhigh throughput rRNA analysis, Nucleic Acids Research 42 (2014) D633D642doi:10.1093/nar/gkt1244.

9. Olsen G. J., Lane D. J., Giovannoni S. J., Pace N.R., Stahl D. A. - Microbial ecology and evolution: a ribosomal RNA approach, Annual review of microbiology 40 (1986) 337365. PMID: 2430518.

10. Sinclair L., Osman O. A., Bertilsson S., Eiler A. - Microbial Community Composition and Diversity via 16S rRNA Gene Amplicons: EvaluatingtheIllumina Platform, PLoS ONE 10 (2) (2015) e0116955.doi:10.1371/journal.pone.0116955.

11. Kozich J. J., Westcott S. L., Baxter N. T., Highlander S. K., Schloss P. D. - Development of a dual-index sequencing strategy and curation pipeline for analyzing amplicon sequence data on the MiSeq Illumina sequencing platform, Appl. Environ. Microbiol.79 (2013) 5112-5120. doi: 10.1128/AEM.01043-13 PMID: 23793624.

12. Sharma P. K., Sharma M., Kaur J. - Metagenomic Approach to Explore Microbial Diversity and Genetic Potential of Uncultured Microorganisms from Different Environment Niches, Res. J. Pharmaceutical, Biological and Chemical Sci. 3 (2) (2012) 947-956. 
13. Rodríguez-Marconi S., De la Iglesia R., DíezB., Fonseca C. A., Hajdu E., Trefault N. Characterization of Bacterial, Archaeal and Eukaryote Symbionts from Antarctic Sponges Reveals a High Diversity at a Three-Domain Level and a Particular Signature for This Ecosystem, PLoS ONE 10 (9) (2015)e0138837. doi:10.1371/journal.pone.0138837.

14. Weidner S., Arnold W., Stackebrandt E., Pu"hler A. - Phylogeneticanalysis of bacterial communities associated with leaves of the sea grass halophilastipulacea by a cultureindependent small-subunit rRNA gene approach, Microbiol. Ecol. 39 (2000) 22- 31.

15. Li Z. Y., He L. M., Wu J., Jiang Q. - Bacterial community diversity associated with four marine sponges from the South China Sea based on 16S rDNA-DGGE finger printing, J. Experimental Marine Biology and Ecology 329 (2006) 75-85.

16. Stieglmeier M., Alves R. J. E., Schleper C. - The Phylum Thaumarchaeota, pp.347-358. In: E. Rosenberg et al. (eds.), The Prokaryotes - Other Major Lineages of Bacteria and the Archaea, DOI 10.1007/978-3-642-38954-2_338,@ Springer-Verlag Berlin Heidelberg 2014.

17. Fan L., Reynolds D., Liu M., Stark M., Kjelleberg S., Webster N. S., and Thomas T. Functional equivalence and evolutionary convergence in complex communities of microbial sponge symbionts PNAS (2012) E1878-E1887 www.pnas.org/cgi/doi/10.1073/pnas.

18. Kennedy J., Flemer B., Jackson S. A., Morrissey J. P., O'Gara F., et al. - Evidence of a Putative Deep Sea Specific Microbiome in Marine Sponges,PLoS ONE 9(3) (2014) e91092. doi:10.1371/journal.pone.0091092.

19. Erwin P.M., Lopez-Legentil S., Gonzalez-Pech R., and Turon X. - A specific mix of generalists: bacterial symbionts in Mediterranean Irciniaspp, FEMS Microbiol. Ecol. 79 (2012): 619-637.

20. Schmitt S., Hentschel U., Taylor M. W. - Deep sequencing reveals diversity and community structure of complex microbiota in five Mediterranean sponges, Hydrobiologia 687(2012)341_351 DOI 10.1007/s10750-011-0799-9.

21. Zeng Y., Feng F., Medová H., Dean J., Koblîek M. - Functional type 2 photosynthetic reaction centers found in the rare bacterial phylum Gemmatimonadetes, Proceedings of the National Academy of Sciences of the United States of America 111 (2014)7795_7800 DOI 10.1073/pnas.1400295111.

22. Olson J. B., Gao X. - Characterizing the bacterial associates of three Caribbean sponges

22. along a gradient from shallow to mesophotic depths, FEMS Microbiol. Ecol. 85 (2013)74_84 DOI 10.1111/1574-6941.12099.

23. Blanquer A., Uriz M.J. and Galand P. E. - Removing environmental sources of variation to gain insight on symbionts vs. transient microbes in high and low microbial abundance sponges. Environmental Microbiology 15 (11) (2013) 3008-3019. 